\title{
Predicting the consistency of intracranial meningiomas using apparent diffusion coefficient maps derived from preoperative diffusion-weighted imaging
}

\author{
Kenya Miyoshi, MD, ${ }^{1}$ Tsukasa Wada, MD, ${ }^{1}$ Ikuko Uwano, PhD, ${ }^{2}$ Makoto Sasaki, MD, ${ }^{2}$ \\ Hiroaki Saura, MD, ${ }^{1}$ Shunrou Fujiwara, PhD, ${ }^{1}$ Fumiaki Takahashi, PhD, ${ }^{3}$ Eiki Tsushima, PhD, ${ }^{4}$ and \\ Kuniaki Ogasawara, MD ${ }^{1}$
}

1'Department of Neurosurgery and ${ }^{2}$ Division of Ultrahigh Field MRI, Institute for Biomedical Sciences, and ${ }^{3}$ Division of Medical Engineering, Department of Information Science, Iwate Medical University School of Medicine, Morioka; and ${ }^{2}$ Department of Physical Therapy, Hirosaki University School of Health Science, Hirosaki, Japan

\begin{abstract}
OBJECTIVE The consistency of meningiomas is a critical factor affecting the difficulty of resection, operative complications, and operative time. The apparent diffusion coefficient (ADC) is derived from diffusion-weighted imaging (DWI) and is calculated using two optimized $b$ values. While the results of comparisons between the standard ADC and the consistency of meningiomas vary, the shifted ADC has been reported to be strongly correlated with liver stiffness. The purpose of the present prospective cohort study was to determine whether preoperative standard and shifted ADC maps predict the consistency of intracranial meningiomas.

METHODS Standard (b values 0 and $1000 \mathrm{sec} / \mathrm{mm}^{2}$ ) and shifted (b values 200 and $1500 \mathrm{sec} / \mathrm{mm}^{2}$ ) ADC maps were calculated using preoperative DWI in patients undergoing resection of intracranial meningiomas. Regions of interest (ROIs) were placed within the tumor on standard and shifted ADC maps and registered on the navigation system. Tumor tissue located at the registered ROI was resected through craniotomy, and its stiffness was measured using a durometer. The cutoff point lying closest to the upper left corner of a receiver operating characteristic (ROC) curve was determined for the detection of tumor stiffness such that an ultrasonic aspirator or scissors was always required for resection. Each tumor tissue sample with stiffness greater than or equal to or less than this cutoff point was defined as hard or soft tumor, respectively.
\end{abstract}

RESULTS For 76 ROls obtained from 25 patients studied, significant negative correlations were observed between stiffness and the standard ADC $(\rho=-0.465, p<0.01)$ and the shifted ADC $(\rho=-0.490, p<0.01)$. The area under the ROC curve for detecting hard tumor (stiffness $\geq 20.8 \mathrm{kPa}$ ) did not differ between the standard ADC $(0.820)$ and the shifted ADC (0.847) $(p=0.39)$. The positive predictive value (PPV) for the combination of a low standard ADC and a low shifted ADC for detecting hard tumor was $89 \%$. The PPV for the combination of a high standard ADC and a high shifted ADC for detecting soft tumor (stiffness $<20.8 \mathrm{kPa}$ ) was $81 \%$.

CONCLUSIONS A combination of standard and shifted ADC maps derived from preoperative DWI can be used to predict the consistency of intracranial meningiomas.

https://thejns.org/doi/abs/10.3171/2020.6.JNS20740

KEYWORDS meningioma; consistency; apparent diffusion coefficient; durometer; diagnostic technique

$\mathrm{M}$ ENINGIOMAS are the most common primary brain tumors, and resection is the first-choice treatment for these tumors. Tumor consistency is a critical factor affecting the difficulty of resection, operative complications, and operative time; softer meningio- mas are reportedly easier to resect and have a lower incidence of operative complications and shorter operative time than stiffer meningiomas. ${ }^{1-4}$ Preoperative prediction of tumor consistency thus allows the selection of an appropriate surgical strategy for resection.

ABBREVIATIONS ADC = apparent diffusion coefficient; $A U C=$ area under the ROC curve; $D W I=$ diffusion-weighted imaging; NPV = negative predictive value; PPV = positive predictive value; $\mathrm{ROC}$ = receiver operating characteristic; $\mathrm{ROI}=$ region of interest; $\mathrm{SPGR}=$ spoiled gradient-recalled acquisition in the steady state. SUBMITTED March 10, 2020. ACCEPTED June 30, 2020. 
Many investigators have assessed meningioma consistency using preoperative MRI. Whereas some studies showed a negative correlation between T2-weighted signal intensity and meningioma consistency, ${ }^{3-8}$ others did not find this correlation. ${ }^{2,9,10}$ Hard meningiomas reportedly had higher fractional anisotropy values on diffusion tensor imaging than soft meningiomas. ${ }^{9-11}$ Furthermore, the results of comparisons between the standard apparent diffusion coefficient (ADC) and the consistency of meningiomas vary: the standard ADC was lower ${ }^{12}$ or higher $^{13}$ in hard meningiomas or was not correlated with meningioma consistency. ${ }^{8}$ The variation in results among these studies may depend on differences in methodological factors. In particular, the accuracy of the subjective grading system of tumor consistency defined based on a difference in devices used for tumor resection ${ }^{12,13}$ or a visual analog scale during surgery ${ }^{8}$ should be verified based on the stiffness of tumor tissue measured objectively using a durometer. ${ }^{14}$ Meningioma consistency is also heterogeneous within a tumor. ${ }^{15}$ The consistency should be determined in each region within a tumor rather than in the whole of a tumor. For this purpose, using a navigation system, regions of interest (ROIs) in preoperative MR images should be matched with locations for resection in an operative field as accurately as possible.

A recent study showed that the shifted ADC calculated using two optimized $b$ values was strongly and negatively correlated with stiffness of the liver in patients with chronic liver diseases. ${ }^{16}$ The standard ADC is calculated as though water molecules move freely, but movement of water molecules is actually limited due to tissue structures such as cell membranes. ${ }^{17}$ The shifted ADC has been devised to weight these limitations. ${ }^{16}$ Shifted ADC maps can be obtained using a conventional MRI system without special or invasive devices.

The purpose of the present prospective cohort study was to determine whether standard and shifted ADC maps derived from preoperative diffusion-weighted imaging (DWI) predict the consistency of intracranial meningiomas.

\section{Methods \\ Patients}

For this prospective observational study, patients were enrolled if they met the following inclusion criteria: intracranial meningioma with a maximum diameter of $\geq 30$ $\mathrm{mm}$ diagnosed on conventional MRI; no previous craniotomy, radiation therapy, or chemotherapy for brain lesions; and age $>16$ years. Patients meeting the following criteria were excluded: no 3-T MRI performed, due to implantable electronic devices or implantable metals, which are contraindications for 3-T MRI; intratumoral hemorrhage on MRI; and informed consent not obtained. Only patients who were diagnosed as having meningiomas by histological examination after resection through craniotomy met the final eligibility requirements for this study.

Our hospital's institutional ethics committee assessed and approved the study protocol. Written, informed consent was obtained from each patient or a family member before enrollment in the study.

\section{Preoperative MRI and Data Analyses}

MRI studies were performed using a 3-T MR scanner (Discovery MR750, GE Healthcare) with an 8-channel head coil within 7 days before surgery. Patients underwent DWI with different $b$ values $(0,200,1000$, and 1500 $\mathrm{sec} / \mathrm{mm}^{2}$ ) based on a single-shot spin-echo echo-planar sequence (repetition time/echo time $=4000 / 72 \mathrm{msec}$; field of view $=240 \mathrm{~mm}^{2}$; matrix $=128 \times 128$; slice thickness $=$ $3 \mathrm{~mm}$ without interslice gaps; motion probing gradient $=3$ orthogonal directions, average $=4$; number of slices $=38$ ). Subsequently, patients underwent gadolinium-enhanced 3D T1-weighted imaging with spoiled gradient-recalled acquisition in the steady state (SPGR) for the navigation system.

Two kinds of ADC maps were calculated using two pairs of diffusion-weighted images obtained with two different b values: a pair of 0 and $1000 \mathrm{sec} / \mathrm{mm}^{2}$ values for the standard ADC map and a pair of 200 and 1500 $\mathrm{sec} / \mathrm{mm}^{2}$ for the shifted ADC map, which was previously reported as the optimal pair of $b$ values for assessing body stiffness. ${ }^{16}$ One to five ROIs, each with a diameter of 10 $\mathrm{mm}$, were placed within homogeneous regions in the tumor on the standard and shifted ADC maps by one neuroradiologist. Locations of ROIs on these two ADC maps were set identically. The ADC value was then determined in each ROI. Furthermore, each ROI set on the standard and shifted ADC maps was registered to the 3D-SPGR images for the navigation system.

\section{Intraoperative Procedures}

After incorporating the 3D-SPGR images including the registered ROIs with a navigation system (Curve, Brainlab AG), the patient underwent craniotomy under general anesthesia. One senior neurosurgeon blinded to the ADC values sharply resected tumor tissue located on the navigation system at a registered ROI with a diameter of $8 \mathrm{~mm}$ without electrical cauterization. Immediately after, to quantitatively assess the consistency of the resected tumor tissue, an investigator blinded to ADC values measured the tumor tissue stiffness as pressure $(\mathrm{kPa})$ using a durometer (NEUTONE TDM-Z2 PM, Try-All Corp.). ${ }^{18,19}$ The device used a presser foot that gauges stiffness by measuring how far the material indents the foot, with a higher pressure indicating a harder material..$^{18,19}$

Furthermore, based on the difference in devices used for tumor resection, the same neurosurgeon classified the consistency of tumor tissues surrounding the ROI into the following 5 grades (defined as device-dependent grades): grade 1 , the tumor is easily removed with only a suction tube; grade 2, the tumor cannot be removed with only a suction tube, and a combination with an ultrasonic aspirator is required; grade 3, an ultrasonic aspirator is required throughout tumor removal; grade 4, the tumor cannot be removed with only an ultrasonic aspirator, and a combination with scissors is required; and grade 5, scissors are required throughout tumor removal.

\section{Statistical Analysis}

Data are expressed as means \pm standard deviations. Spearman's rank correlation coefficient was used to as- 


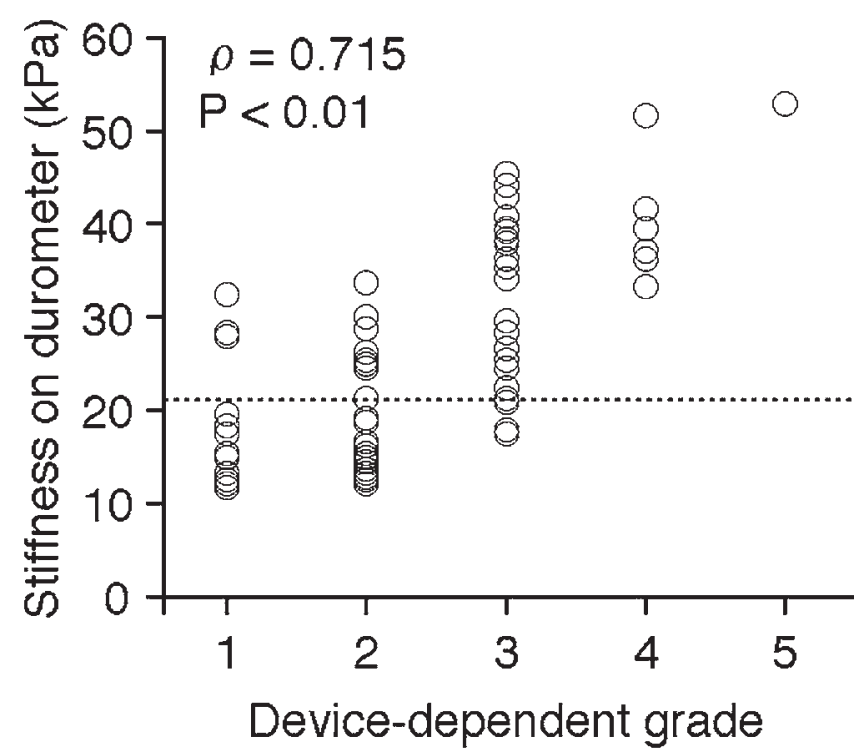

FIG. 1. Relationships between the stiffness of each tumor tissue sample measured using a durometer and the device-dependent grade determined based on differences in the devices used for tumor resection as follows: grade 1, the tumor is easily removed with only a suction tube; grade 2, the tumor cannot be removed with only a suction tube, and a combination with an ultrasonic aspirator is required; grade 3, an ultrasonic aspirator is required throughout tumor removal; grade 4 , the tumor cannot be removed with only an ultrasonic aspirator, and a combination with scissors is required; and grade 5, scissors are required throughout tumor removal. The dotted horizontal line denotes the cutoff point obtained from the ROC curve for the stiffness to detect device-dependent grade $\geq 3$.

sess correlations between two parameters. The difference in these coefficients between two parameters was assessed with the 95\% confidence interval (CI). The cutoff point lying closest to the upper left corner of a receiver operating characteristic (ROC) curve for the stiffness to detect device-dependent grade $\geq 3$ was determined. Each tumor tissue with stiffness $\geq 3$ or $<3$ at this cutoff point was defined as hard or soft tumor, respectively. ROC curves were created to assess the accuracies of the standard ADC and the shifted ADC for detecting hard tumor. The area under the ROC curve (AUC) was used to determine the ability to discriminate between hard tumor and soft tumor. The method of Pepe and Longton ${ }^{20}$ was used for pairwise comparison of the AUCs for the standard ADC and the shifted ADC. A p value $<0.05$ was considered significant for all tests. Differences of sensitivity, specificity, positive predictive values (PPVs), and negative predictive values (NPVs) among the standard ADC, the shifted ADC, and a combination of these two were assessed using 95\% CIs calculated using binomial distributions. Last, taking account for the correlation between different measures on the same tumor, the relationship between the two parameters was evaluated using generalized linear mixed-effects models with a subject random intercept. Device-dependent grade was assumed to be a multinomial variable with a cumulative logit link. Stiffness on a durometer, the standard ADC, and the shifted ADC were assumed to be a normal variable with an identity link. In addition, to elucidate the presence or absence of statistical dependence due to multiple sampling of individual tumors, the ROI population obtained was analyzed using the bootstrap method. Briefly, one ROI from one to five ROIs obtained for each tumor was randomly selected, and samples with different combinations of ROIs were generated. Spearman's rank correlation coefficient and the $\mathrm{p}$ value between two parameters were calculated for each sample. The mean and 95\% CI values of Spearman's rank correlation coefficients and the $\mathrm{p}$ values for all samples were calculated and compared with Spearman's rank correlation coefficients and the $\mathrm{p}$ values for the original ROI population.

\section{Results}

Over the course of 15 months, 29 patients satisfied the inclusion criteria. Four patients were excluded, 3 of whom did not undergo 3-T MRI due to having implantable electronic devices or implantable metals, and 1 patient who had an intratumoral hemorrhage found on MRI. The remaining 25 patients successfully underwent tumor resection through craniotomy and were diagnosed as having meningiomas by histological examination. These patients were finally included in the present study.

The age range of the 25 patients ( 10 men and 15 women) was 41-84 years (mean age $61 \pm 12$ years). The tumor was located at the supratentorial convexity in 7 patients, the parasagittal sinus in 6 patients, the falx cerebri in 3 patients, the tuberculum sellae in 2 patients, the infratentorial convexity in 2 patients, the sphenoid ridge in 2 patients, the olfactory groove in 1 patient, the tentorium cerebelli in 1 patient, and the planum sphenoidale in 1 patient. All meningiomas with Simpson grades $\leq 3$ were successfully resected. Histological examinations diagnosed meningothelial-type meningioma in 13 patients, transitional type in 6 patients, fibrous type in 4 patients, psammomatous type in 1 patient, and clear cell type in 1 patient.

For the 25 patients studied, a total of 78 ROIs were set on MR images. However, the ADC was not obtained due to strong distortion on diffusion-weighted images in the $\mathrm{ROI}$ in the tumor located at the tentorium cerebelli. Tumor tissue in another ROI was not intraoperatively obtained due to the deep location of the targeted ROI. These 2 ROIs were excluded from subsequent analyses, and the remaining 76 ROIs were finally analyzed. Four, $4,9,5$, and 3 patients had 5, 4, 3, 2, and 1 ROIs for analyses, respectively, in each tumor. Overall, 40, 17, 14, 3, and 2 ROIs were meningothelial type, transitional type, fibrous type, clear cell type, and psammomatous type, respectively. The stiffness of the tumor tissue resected in each ROI was successfully measured using a durometer in all 76 ROIs. Tumor tissues surrounding 17, 28, 24, 6, and 1 ROIs were intraoperatively classified into device-dependent grades 1, 2, 3, 4, and 5, respectively. Thirteen (59\%) of 22 patients with $\geq 2$ ROIs in a tumor had ROIs with different device-dependent grades in the tumor.

A significant positive correlation was observed between the device-dependent grade and the stiffness of each tumor tissue measured using a durometer $(\rho=0.715$, 95\% CI 0.584-0.810; $\mathrm{p}<0.01$ ) (Fig. 1). The cutoff point lying closest to the upper left corner of the ROC curve for 

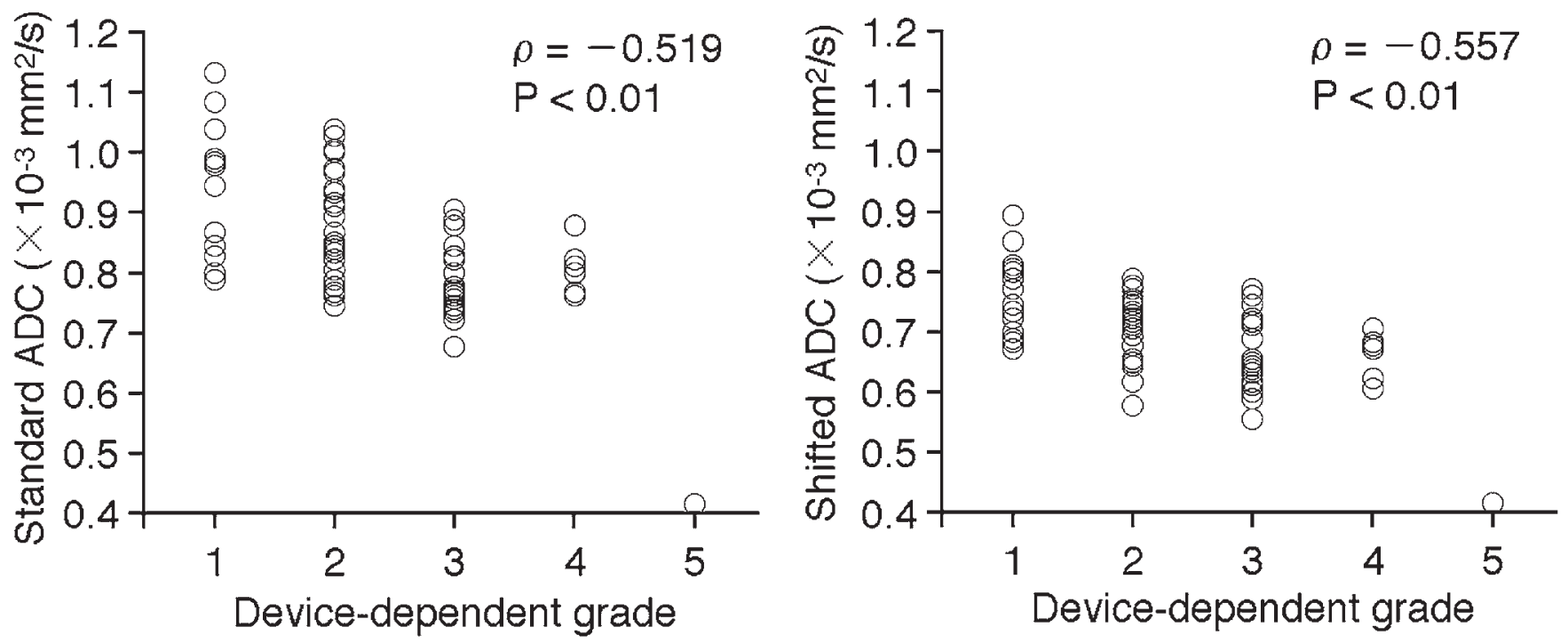

FIG. 2. Relationships between device-dependent grade and the standard ADC (left) or the shifted ADC (right).

the stiffness to detect a device-dependent grade $\geq 3$ was $20.8 \mathrm{kPa}$ (Fig. 1). With that cutoff point, the sensitivity, specificity, PPV, and NPV were $90 \%, 76 \%, 72 \%$, and $92 \%$, respectively. Thus, 40 tumor tissues with stiffness $\geq 20.8$ $\mathrm{kPa}$ and 36 tumor tissues with stiffness $<20.8 \mathrm{kPa}$ were defined as hard and soft tumors, respectively.

Significant negative correlations were observed between the device-dependent grade and the standard ADC $(\rho=-0.519,95 \% \mathrm{CI}-0.667$ to $-0.333 ; p<0.01)$ and the shifted ADC $(\rho=-0.557,95 \%$ CI -0.695 to $-0.379 ; p<$ 0.01) (Fig. 2). The correlation coefficients did not differ between these two ADCs. Significant negative correlations were observed between the stiffness and the standard $\operatorname{ADC}(\rho=-0.465,95 \% \mathrm{CI}-0.624$ to $-0.267 ; \mathrm{p}<0.01)$ and the shifted ADC $(\rho=-0.490,95 \%$ CI -0.645 to -0.298 ; p < 0.01) (Fig. 3). The correlation coefficients also did not differ between these two ADCs.
Based on Fig. 3, which shows the relationships among the standard ADC, shifted ADC, and stiffness, ROC curves for the former two for detecting hard tumor (stiffness $\geq 20.8 \mathrm{kPa}$ ) were calculated as shown in Fig. 4. AUCs for the standard ADC and the shifted ADC were 0.820 (95\% CI 0.716-0.899) and 0.847 (95\% CI 0.746-0.919), respectively. The AUCs did not differ between the standard ADC and the shifted ADC (difference between AUCs, $0.027 ; p=0.39$ ). Table 1 shows the sensitivity, specificity, PPV, and NPV for the standard and shifted ADCs with the cutoff point lying closest to the upper left corner of the ROC curve for detecting hard tumor. The sensitivity and NPV were greater for the standard ADC than for the shifted ADC, and the specificity and PPV were greater for the shifted ADC than for the standard ADC, though a significant difference between these two ADCs was observed only for specificity.
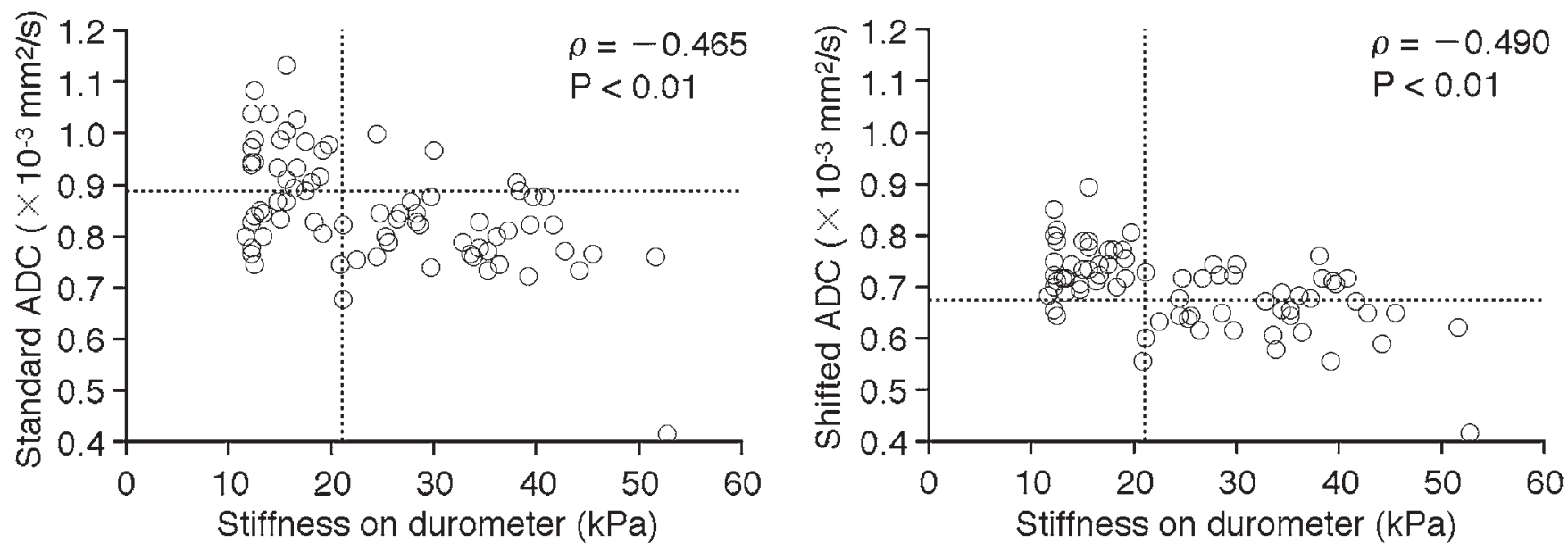

FIG. 3. Relationships between stiffness on a durometer and the standard ADC (left) or the shifted ADC (right). The dotted vertical lines denote the cutoff points obtained from ROC curves for the stiffness used to detect device-dependent grade $\geq 3$. The dotted horizontal lines denote the cutoff points obtained from the ROC curves for the hard tumor defined based on the stiffness. 


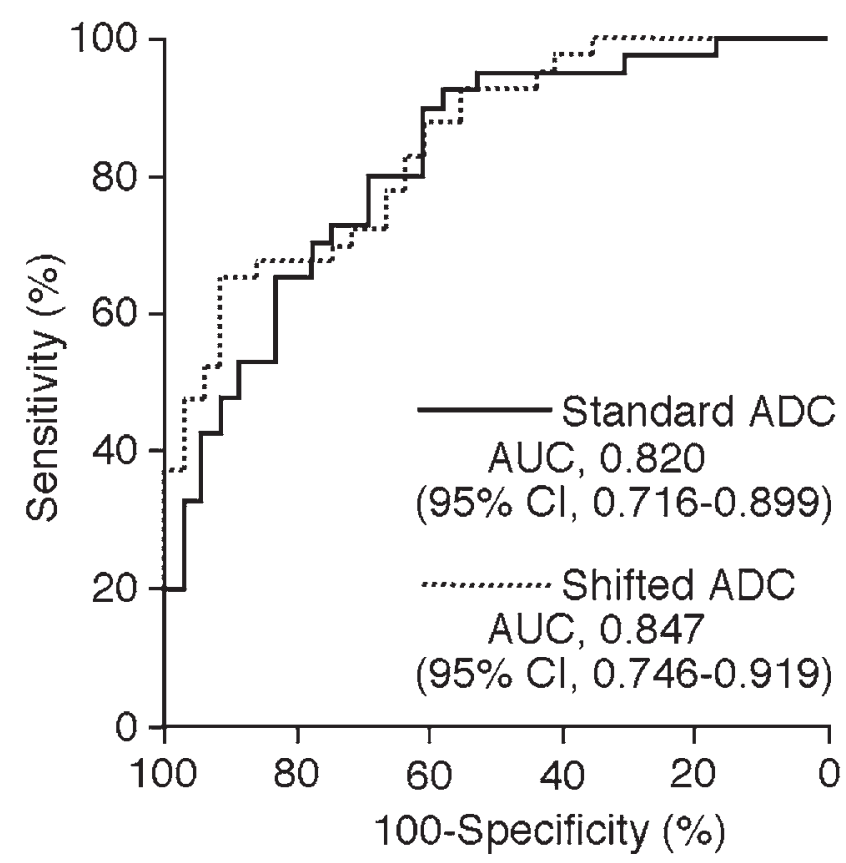

FIG. 4. ROC curves used to compare accuracy between the standard $A D C$ and the shifted ADC for the detection of hard tumor.

Figure 5 shows the relationships among the standard ADC, the shifted ADC, and the consistency of the tumor tissue. A strong positive correlation was observed between the former two $(\rho=0.894,95 \%$ CI $0.837-0.932$; $p<0.01)$. The PPV for the combination of standard ADC $\leq 0.880 \times$ $10^{-3}$ and shifted ADC $\leq 0.680 \times 10^{-3}$ for detecting hard tumor was $89 \%$ (95\% CI 77\%-100\%). The PPV for the combination of standard ADC $>0.880 \times 10^{-3}$ and shifted ADC $>0.680 \times 10^{-3}$ for detecting soft tumor was $81 \%(95 \% \mathrm{CI}$ $67 \%-96 \%)$. The PPV for the combination of standard ADC $\leq 0.880 \times 10^{-3}$ and shifted ADC $>0.680 \times 10^{-3}$ for detecting hard tumor was $50 \%(95 \%$ CI $29 \%-71 \%)$. There was only one ROI with a combination of conventional ADC > $0.880 \times 10^{-3}$ and shifted ADC $\leq 0.680 \times 10^{-3}$.

Gadolinium-enhanced 3D T1-weighted SPGR images and standard and shifted ADC maps in each patient with hard or soft meningiomas are shown in Fig. 6.

Generalized linear mixed-effects models with a subject random intercept showed a significant relationship between the device-dependent grade and the stiffness on a durometer $(\mathrm{p}<0.01)$, between the device-dependent grade and the standard ADC ( $\mathrm{p}<0.01)$, between the device-dependent grade and the shifted ADC $(\mathrm{p}<0.01)$, between the stiffness on a durometer and the standard ADC $(\mathrm{p}<$ 0.01 ), and between the stiffness on a durometer and the shifted ADC $(\mathrm{p}<0.01)$.

When the original ROI population (76 ROIs) was analyzed using the bootstrap method, the total number of samples with different combinations of 25 ROIs (25 tumors in total) was theoretically $>50$ billion $\left(5^{4} \times 4^{4} \times 3^{9}\right.$ $\times 2^{5} \times 1^{3}$ ). Therefore, just 10,000 samples with different combinations of 25 ROIs were randomly selected and analyzed (Table 2). Spearman's rank correlation coefficients and the $\mathrm{p}$ values between any two parameters for the original ROI population were approximately equal to the mean and $95 \%$ CI of such values obtained from 10,000 samples.

\section{Discussion}

The present study demonstrated that a combination of standard and shifted ADC maps derived from preoperative DWI predicts the consistency of intracranial meningiomas.

ROIs $\geq 2$ were obtained in 22 of 25 tumors studied. This multiple sampling of individual tumors might cause statistical overestimation or dependency. Therefore, taking into account the correlation between different measures on the same tumor, the relationship between the two parameters was evaluated using generalized linear mixed-effects models with a subject random intercept. As a result, significant relationships between both parameters were observed, suggesting the absence of statistical overestimation caused by the multiple sampling. Further, the bootstrap method was used to determine the presence or absence of statistical dependency in our original ROI population (76 ROIs). It was found that Spearman's rank correlation coefficients and the $\mathrm{p}$ values between any two parameters for the original ROI population were approximately equal to the mean and $95 \%$ CI of such values obtained from the 10,000 samples. These finding suggest the absence of statistical dependency in the original ROI population.

Meningioma consistency is heterogeneous within a tumor. ${ }^{15}$ Actually, in the present study, more than half of the patients with ROIs $\geq 2$ within a tumor had ROIs showing different device-dependent grades within the tumor. The correlation coefficient between the subjective device-dependent grading system and the stiffness of tumor tissue measured using a durometer corresponded with that re-

TABLE 1. Sensitivity, specificity, PPV, and NPV for the standard ADC and the shifted ADC for the detection of hard meningiomas

\begin{tabular}{lccc}
\hline & $\begin{array}{c}\text { Standard } \\
\text { ADC }\end{array}$ & $\begin{array}{c}\text { Shifted } \\
\text { ADC }\end{array}$ & $\begin{array}{c}\text { Statistical Significance } \\
\text { (by comparison of 95\% Cls) }\end{array}$ \\
\hline Sensitivity $(95 \% \mathrm{Cl})$ & $88 \%(77-98 \%)$ & $63 \%(47-78 \%)$ & No \\
\hline Specificity $(95 \% \mathrm{Cl})$ & $61 \%(45-77 \%)$ & $92 \%(83-100 \%)$ & Yes \\
\hline PPV $(95 \% \mathrm{Cl})$ & $71 \%(59-84 \%)$ & $89 \%(78-100 \%)$ & No \\
\hline NPV $(95 \% \mathrm{Cl})$ & $81 \%(67-96 \%)$ & $69 \%(56-82 \%)$ & No \\
\hline Cutoff point $\left(\mathrm{mm}^{2} / \mathrm{sec}\right)$ & $0.880 \times 10^{-3}$ & $0.680 \times 10^{-3}$ & \\
\hline
\end{tabular}




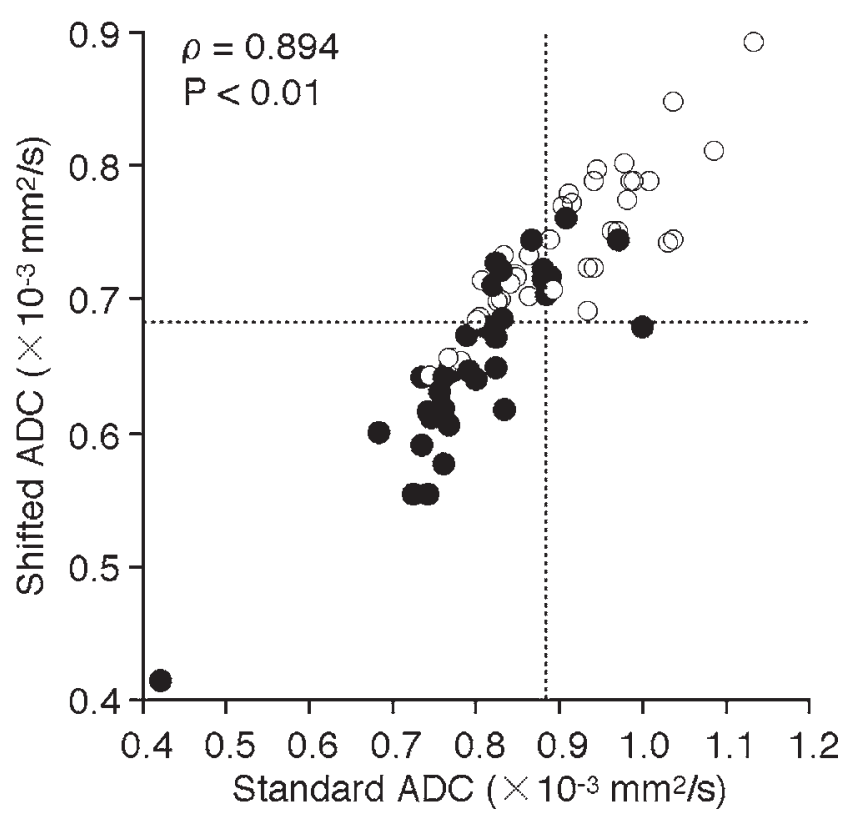

FIG. 5. Relationships among the standard ADC, the shifted ADC, and the consistency of tumor tissue. Closed and open circles denote hard and soft tumor tissues, respectively. The dotted horizontal and vertical lines denote the cutoff points obtained from the ROC curves for discriminating between hard and soft tumor tissues defined based on the stiffness. ported in a previous study. ${ }^{14}$ In addition, using a navigation system, ROIs in preoperative MR images were matched with locations for resection on an operative field as accurately as possible. Thus, the standard ADC and shifted ADC values were negatively correlated with the devicedependent grade or the stiffness of tumor tissue measured using a durometer.

MR elastography can obtain a body stiffness map. Several studies have demonstrated that stiffness on MR elastography was correlated more strongly with meningioma consistency than T1- or T2-weighted signal intensity. ${ }^{2,14}$ However, because stiffness on MR elastography is calculated based on the phase shift made by intentional mechanical vibration obtained from data with and without this vibration, this method needs a special device to apply vibration to the brain., ${ }^{214}$ Whether the mechanical vibration affects the brain also remains unknown. The correlation coefficient of the shifted ADC was approximately equal to that between stiffness on this MR elastography and the device-dependent grade or the stiffness measured using a durometer. ${ }^{14}$ Thus, the shifted ADC may predict the consistency of meningioma with the same accuracy as MR elastography.

Because ADC is a continuous variable, hard or soft tumor was determined based on stiffness as a continuous variable to assess the accuracy of the standard ADC or the shifted ADC for discrimination between hard tumor and soft tumor. For this determination, a hard tumor was clinically defined as requiring an ultrasonic aspirator or

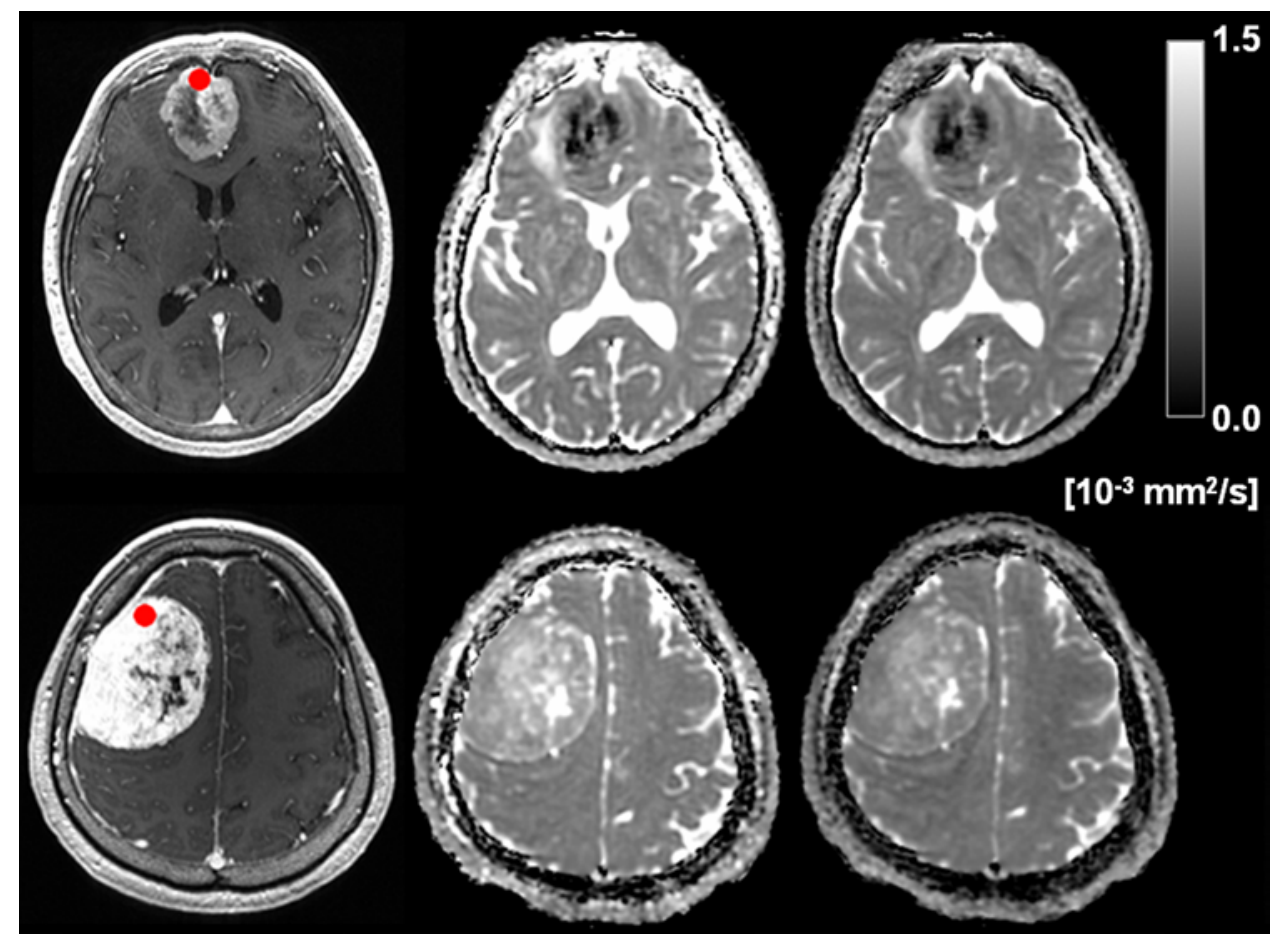

FIG. 6. Gadolinium-enhanced 3D T1-weighted images with SPGR (left column), standard (middle column), and shifted (right column) ADC maps in a 68-year-old woman (upper row) and a 49-year-old man (lower row) with a hard and soft meningioma, respectively. Red closed circles denote ROls placed within the tumor. The ADC values on both standard and shifted ADC maps are decreased in the hard meningioma and increased in the soft meningioma compared with the cerebral tissue contralateral to the tumor. 
TABLE 2. Spearman's rank correlation coefficients and $p$ values between any two parameters from the original ROI population (76 ROIs) and the means and $95 \% \mathrm{Cls}$ of such values obtained from 10,000 samples with different combinations of 25 ROls

\begin{tabular}{|c|c|c|c|c|c|c|c|c|}
\hline & \multicolumn{4}{|c|}{ Spearman's Rank Correlation Coefficient } & \multicolumn{4}{|c|}{ Spearman's Rank Correlation Coefficient $p$ Value } \\
\hline & \multicolumn{3}{|c|}{$\begin{array}{c}10,000 \text { Samples w/ Different } \\
\text { Combinations of } 25 \text { ROls }\end{array}$} & \multirow{3}{*}{$\begin{array}{c}\text { Original ROI } \\
\text { Population } \\
\text { (76 ROls) }\end{array}$} & \multicolumn{3}{|c|}{$\begin{array}{l}\text { 10,000 Samples w/ Different } \\
\text { Combinations of } 25 \text { ROls }\end{array}$} & \multirow{3}{*}{$\begin{array}{c}\text { Original RO } \\
\text { Population } \\
\text { (76 ROls) }\end{array}$} \\
\hline & \multirow[b]{2}{*}{ Mean } & \multicolumn{2}{|c|}{$95 \% \mathrm{Cl}$} & & \multirow[b]{2}{*}{ Mean } & \multicolumn{2}{|c|}{$95 \% \mathrm{Cl}$} & \\
\hline & & Lower Limit & Upper Limit & & & Lower Limit & Upper Limit & \\
\hline $\begin{array}{l}\text { Device-dependent grade and } \\
\text { stiffness on durometer }\end{array}$ & 0.746 & 0.745 & 0.746 & 0.715 & $<0.01$ & $<0.01$ & $<0.01$ & $<0.01$ \\
\hline $\begin{array}{l}\text { Device-dependent grade and } \\
\text { standard ADC }\end{array}$ & -0.600 & -0.605 & -0.599 & -0.519 & $<0.01$ & $<0.01$ & $<0.01$ & $<0.01$ \\
\hline $\begin{array}{l}\text { Device-dependent grade and } \\
\text { shifted ADC }\end{array}$ & -0.633 & -0.633 & -0.636 & -0.557 & $<0.01$ & $<0.01$ & $<0.01$ & $<0.01$ \\
\hline $\begin{array}{l}\text { Stiffness on durometer and } \\
\text { standard ADC }\end{array}$ & -0.537 & -0.538 & -0.537 & -0.465 & 0.01 & 0.01 & 0.01 & $<0.01$ \\
\hline $\begin{array}{l}\text { Stiffness on durometer and } \\
\text { shifted ADC }\end{array}$ & -0.578 & -0.578 & -0.577 & -0.490 & $<0.01$ & $<0.01$ & $<0.01$ & $<0.01$ \\
\hline
\end{tabular}

scissors throughout tumor removal. Based on the ROC analysis, a hard tumor was defined as having stiffness $\geq$ $20.8 \mathrm{kPa}$. This value was considerably greater than the value of $>6 \mathrm{kPa}$ used in a study of MR elastography. ${ }^{14}$ However, that study did not explain the reason for using that definition. Our subsequent analysis demonstrated that the standard ADC or the shifted ADC discriminated between hard tumor and soft tumor with moderate accuracy, and this discriminative ability did not differ between the standard ADC and the shifted ADC. It also showed that approximately $90 \%$ of hard tumors or soft tumors showed a low standard ADC or a high shifted ADC, respectively, but the PPV for detecting hard tumor or soft tumor differed among combinations of the standard ADC and the shifted ADC. Overall, $\geq 80 \%$ of tumors with a low standard ADC and a low shifted ADC were hard tumors, and $\geq 80 \%$ of tumors with a high standard ADC and a high shifted ADC were soft tumors. These findings suggest the need to prepare an ultrasonic aspirator and/or scissors for resection of tumors with a low standard ADC and a low shifted ADC. In particular, when the endoscopic endonasal skull base approach for resection of anterior skull base meningiomas is planned, the combination of these two ADCs gives important information. In contrast, half of the tumors with a low standard ADC and a high shifted ADC were soft, and the other half were hard. Thus, consistency of tumors with this condition might be unpredictable, which is a limitation of the present method.

Consistency of a meningioma generally depends on the fibrous component content and the cell density. ${ }^{21,22} \mathrm{~A}$ hard tumor is rich in fibrous components and has high cell density that results in strong intercellular adhesion. ${ }^{22}$ While the standard ADC correlates with tumor cellularity and the amount of fibrous tissue, ${ }^{23-25}$ it is also affected by intravoxel incoherent motion and non-Gaussian diffusion. ${ }^{26,27}$ In an intravoxel incoherent motion model, biological tissue contains two distinct environments: molecular diffusion of water in the tissue (sometimes referred to as "true diffusion") and microcirculation of blood in the capillary net- work (perfusion). ${ }^{26,27}$ The latter reportedly contributes to the standard ADC. ${ }^{4,12}$ Furthermore, the standard ADC is calculated as water molecules move according to Gaussian (normal) diffusion, and the signal decays monoexponentially. ${ }^{16}$ However, these conditions are actually limited in biological tissue. The shifted ADC is calculated by using two key b values optimized to reflect Gaussian diffusion, non-Gaussian diffusion, and intravoxel incoherent motion. ${ }^{16}$ The finding that specificity and the PPV for detecting hard tumor were greater for the shifted ADC than for the standard ADC suggested that soft tumor is excessively affected by tumor cellularity and the amount of fibrous tissue. In contrast, the finding that sensitivity and the NPV for detecting hard tumor were greater for the standard ADC than for the shifted ADC suggested that hard tumor is affected by microcirculation of blood in the tumor, as well as tumor cellularity and the amount of fibrous tissue.

A pair of b values (200 and $1500 \mathrm{sec} / \mathrm{mm}^{2}$ ) was used for the shifted ADC map. This was previously reported as the optimal pair of $b$ values for assessing body stiffness. ${ }^{16}$ The optimal combination of $b$ values is theoretically organ specific (e.g., body vs brain) but, according to the existing literature, it should be very similar for all human tissues. ${ }^{16}$

As a limitation other than the combinations of the standard ADC and the shifted ADC mentioned above, the ADC was not obtained due to strong distortion on diffusion-weighted images in an ROI in the tumor located at the tentorium cerebelli. Magnetic susceptibility differences among brain tissue, bone, and air-filled sinuses cause geometrical distortion in MR images, in particular, diffusionweighted images. ${ }^{28}$ ADC maps may not be generated under such conditions.

\section{Conclusions}

The present study demonstrated that a combination of standard and shifted ADC maps derived from preoperative DWI predicts the consistency of intracranial meningiomas. 


\section{Acknowledgments}

This work was partly supported by Grants-in-Aid for Strategic Medical Science Research from the Ministry of Education, Culture, Sports, Science and Technology of Japan (S1491001) and for Scientific Research from the Japan Society for the Promotion of Science (JP18K09002).

\section{References}

1. Jääskeläinen J. Seemingly complete removal of histologically benign intracranial meningioma: late recurrence rate and factors predicting recurrence in 657 patients. A multivariate analysis. Surg Neurol. 1986;26(5):461-469.

2. Murphy MC, Huston J III, Glaser KJ, et al. Preoperative assessment of meningioma stiffness using magnetic resonance elastography. J Neurosurg. 2013;118(3):643-648.

3. Sitthinamsuwan B, Khampalikit I, Nunta-aree $S$, et al. Predictors of meningioma consistency: a study in 243 consecutive cases. Acta Neurochir (Wien). 2012;154(8):1383-1389.

4. Yao A, Pain M, Balchandani P, Shrivastava RK. Can MRI predict meningioma consistency?: a correlation with tumor pathology and systematic review. Neurosurg Rev. 2018;41(3): $745-753$.

5. Hoover JM, Morris JM, Meyer FB. Use of preoperative magnetic resonance imaging $\mathrm{T} 1$ and $\mathrm{T} 2$ sequences to determine intraoperative meningioma consistency. Surg Neurol Int. 2011;2:142.

6. Ortega-Porcayo LA, Ballesteros-Zebadúa P, Marrufo-Meléndez OR, et al. Prediction of mechanical properties and subjective consistency of meningiomas using T1-T2 assessment versus fractional anisotropy. World Neurosurg. 2015;84(6): 1691-1698.

7. Smith KA, Leever JD, Chamoun RB. Predicting consistency of meningioma by magnetic resonance imaging. $J$ Neurol Surg B Skull Base. 2015;76(3):225-229.

8. Watanabe K, Kakeda S, Yamamoto J, et al. Prediction of hard meningiomas: quantitative evaluation based on the magnetic resonance signal intensity. Acta Radiol. 2016;57(3):333-340.

9. Kashimura H, Inoue T, Ogasawara K, et al. Prediction of meningioma consistency using fractional anisotropy value measured by magnetic resonance imaging. J Neurosurg. 2007; 107(4):784-787.

10. Romani R, Tang WJ, Mao Y, et al. Diffusion tensor magnetic resonance imaging for predicting the consistency of intracranial meningiomas. Acta Neurochir (Wien). 2014;156(10): 1837-1845.

11. Tropine A, Dellani PD, Glaser M, et al. Differentiation of fibroblastic meningiomas from other benign subtypes using diffusion tensor imaging. J Magn Reson Imaging. 2007;25(4): 703-708.

12. Yogi A, Koga T, Azama K, et al. Usefulness of the apparent diffusion coefficient (ADC) for predicting the consistency of intracranial meningiomas. Clin Imaging. 2014;38(6):802-807.

13. Phuttharak W, Boonrod A, Thammaroj J, et al. Preoperative MRI evaluation of meningioma consistency: a focus on detailed architectures. Clin Neurol Neurosurg. 2018;169: $178-184$

14. Hughes JD, Fattahi N, Van Gompel J, et al. Higher-resolution magnetic resonance elastography in meningiomas to determine intratumoral consistency. Neurosurgery. 2015;77(4): 653-659.

15. Zada G, Yashar P, Robison A, et al. A proposed grading system for standardizing tumor consistency of intracranial meningiomas. Neurosurg Focus. 2013;35(6):E1.
16. Le Bihan D, Ichikawa S, Motosugi U. Diffusion and intravoxel incoherent motion MR imaging-based virtual elastography: a hypothesis-generating study in the liver. Radiology. 2017;285(2):609-619.

17. Iima M, Kataoka M, Kanao S, et al. Intravoxel incoherent motion and quantitative non-gaussian diffusion MR imaging: evaluation of the diagnostic and prognostic value of several markers of malignant and benign breast lesions. Radiology. 2018;287(2):432-441.

18. Uchiyama T, Nagaoka M. Durometer. Article in Japanese. Biomechanisms Japan. 2016;40:97-102.

19. Kogo H, Miyabara H, Okawa H, et al. Effect of body flexibility on the lumbar muscle stiffness of community-dwelling elderly. Rigakuryoho Kagaku. 2015;30(4):605-608.

20. Pepe MS, Longton G. Standardizing diagnostic markers to evaluate and compare their performance. Epidemiology. 2005;16(5):598-603.

21. Maiuri F, Iaconetta G, de Divitiis O, et al. Intracranial meningiomas: correlations between MR imaging and histology. Eur J Radiol. 1999;31(1):69-75.

22. Muthupillai R, Rossman PJ, Lomas DJ, et al. Magnetic resonance imaging of transverse acoustic strain waves. Magn Reson Med. 1996;36(2):266-274.

23. Castillo M, Smith JK, Kwock L, Wilber K. Apparent diffusion coefficients in the evaluation of high-grade cerebral gliomas. AJNR Am J Neuroradiol. 2001;22(1):60-64.

24. Gauvain KM, McKinstry RC, Mukherjee P, et al. Evaluating pediatric brain tumor cellularity with diffusion-tensor imaging. AJR Am J Roentgenol. 2001;177(2):449-454.

25. Guo AC, Cummings TJ, Dash RC, Provenzale JM. Lymphomas and high-grade astrocytomas: comparison of water diffusibility and histologic characteristics. Radiology. 2002; 224(1):177-183.

26. Le Bihan D, Breton E, Lallemand D, et al. Separation of diffusion and perfusion in intravoxel incoherent motion MR imaging. Radiology. 1988;168(2):497-505.

27. Le Bihan D, Breton E, Lallemand D, et al. MR imaging of intravoxel incoherent motions: application to diffusion and perfusion in neurologic disorders. Radiology. 1986;161(2): 401-407.

28. Le Bihan D, Poupon C, Amadon A, Lethimonnier F. Artifacts and pitfalls in diffusion MRI. J Magn Reson Imaging. 2006;24(3):478-488.

\section{Disclosures}

Dr. Ogasawara reports receiving a consigned research fund from Nihon Medi-Physics Co., Ltd.

\section{Author Contributions}

Conception and design: Ogasawara, Wada. Acquisition of data: Miyoshi, Uwano, Saura. Analysis and interpretation of data: Ogasawara, Miyoshi, Uwano, Saura. Drafting the article: Ogasawara, Miyoshi. Critically revising the article: Sasaki, Fujiwara. Reviewed submitted version of manuscript: Fujiwara. Approved the final version of the manuscript on behalf of all authors: Ogasawara. Statistical analysis: Fujiwara, Takahashi, Tsushima. Study supervision: Wada, Sasaki.

\section{Correspondence}

Kuniaki Ogasawara: Iwate Medical University, Morioka, Japan. kuogasa@iwate-med.ac.jp. 